\title{
Which process metrics can significantly improve defect prediction models? An empirical study
}

\author{
Lech Madeyski • Marian Jureczko
}

Published online: 17 June 2014

(C) The Author(s) 2014. This article is published with open access at Springerlink.com

\begin{abstract}
The knowledge about the software metrics which serve as defect indicators is vital for the efficient allocation of resources for quality assurance. It is the process metrics, although sometimes difficult to collect, which have recently become popular with regard to defect prediction. However, in order to identify rightly the process metrics which are actually worth collecting, we need the evidence validating their ability to improve the product metric-based defect prediction models. This paper presents an empirical evaluation in which several process metrics were investigated in order to identify the ones which significantly improve the defect prediction models based on product metrics. Data from a wide range of software projects (both, industrial and open source) were collected. The predictions of the models that use only product metrics (simple models) were compared with the predictions of the models which used product metrics, as well as one of the process metrics under scrutiny (advanced models). To decide whether the improvements were significant or not, statistical tests were performed and effect sizes were calculated. The advanced defect prediction models trained on a data set containing product metrics and additionally Number of Distinct Committers (NDC) were significantly better than the simple models without NDC, while the effect size was medium and the probability of superiority (PS) of the advanced models over simple ones was high $(p=.016, r=-.29$, $\mathrm{PS}=.76$ ), which is a substantial finding useful in defect prediction. A similar result with slightly smaller PS was achieved by the advanced models trained on a data set containing product metrics and additionally all of the investigated process metrics $(p=.038$, $r=-.29$, PS $=.68$ ). The advanced models trained on a data set containing product metrics and additionally Number of Modified Lines (NML) were significantly better than the simple models without NML, but the effect size was small $(p=.038, r=.06)$. Hence,
\end{abstract}

\footnotetext{
L. Madeyski $(\bowtie) \cdot$ M. Jureczko

Wroclaw University of Technology, Wyb.Wyspianskiego 27, 50370 Wrocław, Poland

e-mail: Lech.Madeyski@pwr.edu.pl

URL: http://madeyski.e-informatyka.pl/

M. Jureczko

e-mail: Marian.Jureczko@pwr.edu.pl
} 
it is reasonable to recommend the NDC process metric in building the defect prediction models.

Keywords Software metrics · Product metrics - Process metrics · Defect prediction models $\cdot$ Software defect prediction

\section{Introduction}

Software development companies are seeking for ways to improve the quality of software systems without allocating too many resources in the quality assurance activities such as testing. Applying the same testing effort to all modules of a software system is not an optimal approach, since the distribution of defects among individual parts of a system is not uniform. According to Pareto-Zipf-type law (Boehm and Papaccio 1988; Denaro and Pezzè 2002; Endres and Rombach 2003), the 80:20 empirical rule is operating here, i.e., a small amount of code (often quantified as $20 \%$ of the code) is responsible for the majority of software faults (often quantified as $80 \%$ of the faults). Therefore, it is possible to test only a small part of a software system and find most of the defects. Defect prediction models, in turn, may be used to find the defect-prone classes. Hence, the quality assurance efforts should be focused (unless for critical projects) on the most defect-prone classes in order to save valuable time and financial resources, and, at the same time, to increase the quality of delivered software products.

The defect prediction models built on the basis of product metrics are already well known (Basili et al. 1996; Denaro and Pezzè 2002; Gyimothy et al. 2005; Tang et al. 1999); however, also the process metrics have recently become popular ${ }^{1}$. Fenton was not only among the first who have criticized the product metric-based approach (Fenton and Ohlsson 2000), but also the one who suggested a model based only on the project and the process metrics (Fenton et al. 2007). There are also other studies in which the process metrics are investigated (Illes-Seifert and Paech 2010; Schröter et al. 2006), as well as used in the model (Graves et al. 2000; Weyuker et al. 2008, 2010). Nevertheless, there are no conclusive results. Usually, only the correlations between some process metrics and the defect count are investigated, e.g. (Illes-Seifert and Paech 2010; Schröter et al. 2006). When defect prediction models are built, they are either not compared with a productbased approach (e.g., Bell et al. 2006; Hassan 2009; Ostrand et al. 2005; Weyuker et al. 2006, 2007), they are built on a small sample (e.g., Graves et al. 2000; Moser et al. 2008) or do not perform statistical tests and effect size calculations to conclude whether the improvements obtained through adding the process metrics were of both, statistical and practical significance even when improvements were impressive (e.g., Nagappan et al. 2008). Effect size is an index that quantifies the degree of practical significance of study results, i.e., the degree to which the study results should be considered important, or negligible, regardless of the size of the study sample. Further discussion of related work is given in detail in Sect. 3.

\footnotetext{
${ }^{1}$ In discerning between the two metric types, we follow the Henderson-Sellers' (1996) definitions of product and process metrics (product metric refers to software "snapshot" at a particular point of time, while process metrics reflects the changes over time, e.g., the number of code changes). Even though recently the term "historical metrics" has been used with growing frequency to replace the "process metrics," e.g. (IllesSeifert and Paech 2010), we decided to use the traditional nomenclature.
} 
This paper presents the results of an empirical study exploring the relationship between the process metrics and the number of defects. For that purpose, the correlations between particular process metrics and the number of defects were calculated. Subsequently, the simple defect prediction models were built on the basis of the product metrics. With those simple models, we were able to build advanced defect prediction models by introducing, additionally, one of the process metrics at a time. As a result, we were able to compare the simple and the advanced models and answer the question whether or not the introduction of the selected process metric improved the adequacy of the predictions. Statistical methods were used to evaluate the significance of that improvement. The approach used in this study can be easily put into practice, which is its distinct advantage. Moreover, no sophisticated methods were used to build the prediction models, but the ordinary stepwise linear regression. Even though they are probably neither best nor the most effective for this purpose, stepwise linear regression methods are widely known and, therefore, reduce the learning effort.

The derivation of the baseline model, as well as the experiments presented in this paper, intend to reflect the industrial reality. Since the product metrics have a very long history (e.g., McCabe 1976), they enjoy a good tool support (e.g., the Ckjm tool used in this study) and are well understood by practitioners. We may assume that there are companies interested in defect prediction which have already launched a metric program and collect the product metrics. The assumption is plausible, as such companies are already known to the authors of this paper. A hypothetical company as described above is using product metrics for the aforementioned reasons (mainly tool support). Unfortunately, the prediction results are often unsatisfactory; therefore, new metrics may be employed in order to improve the prediction. The process metrics can be particularly useful, since they reflect the attributes different from those associated with the product metrics, namely the product history, which is (hopefully) an extra source of information. Nevertheless, it is still not obvious what the company should do, as there are a number of process metrics which are being investigated with regard to defect prediction. Furthermore, the results are sometimes contradictory (see Sect. 3 for details). Moreover, the tool support for the process metrics is far from being perfect, e.g., for the sake of this study, the authors had to develop their own solution to calculate these metrics. Bearing in mind that hypothetical situation in an industrial environment and relying on their direct and indirect experience, the authors of this study chose as its main objective to provide assistance in making key decisions regarding which metric (or metrics) should be chosen and added to the metric program in order to improve the predictions and not to waste financial resources on checking all the possibilities. Therefore, we have analyzed which of the frequently used process metrics can significantly improve defect prediction - on the basis of a wide range of software projects from different environments. The construction of the models made use solely of the data which were historically older than the ones used in prediction (model evaluation). For example, the model built on the data from the release $i$ was used to make predictions in release $i+1$. The data from $i$ th release are usually (or at least can be) available during the development of $(i+1)$ th release. Hopefully, on the basis of the empirical evaluations presented in this paper, development teams may take informed decisions (at least to some extent, as the number of analyzed projects, although large, is not infinite) about the process metrics which may be worth collecting in order to improve the defect prediction models based on product metrics. Additionally, the framework of the empirical evaluation of the models presented in this paper can be reused in different environments to evaluate new kinds of metrics and to improve the defect prediction models even further. 
This paper is organized as follows: The descriptions of all the investigated product and process metrics, as well as the tools employed for data collection and the investigated software projects are described in Sect. 2. Related empirical studies concerning the process metrics are presented in Sect. 3. Section 4 contains the detailed description of our empirical investigation aimed at identifying the process metrics which may significantly improve the defect prediction models based on the product metrics. The obtained results are reported in Sect. 5, while threats to validity are discussed in Sect. 6. The discussion of results in Sect. 7 is followed by the conclusions and contributions in Sect. 8 .

\section{Data collection}

This section presents the descriptions of all the investigated product and process metrics in Sect. 2.1, the tools used to compute the aforementioned metrics are described in Sect. 2.2, while the investigated software projects are presented in Sect. 2.3.

\subsection{Studied metrics}

The investigation entailed two types of metrics: the product metrics, which describe the size and design complexity of software, served as the basis and the point of departure, whereas the process metrics were treated as the primary object of this study. The product metrics were used to build simple defect prediction models, while the product metrics, together with the selected process metrics (one at a time), were used to build the advanced models. Subsequently, both models were compared in order to determine whether the selected process metrics improve the prediction efficiency. The classification of the product and the process metrics was thoroughly discussed in (Henderson-Sellers 1996).

\subsubsection{Product metrics}

The following metrics have been used in this study:

- The metrics suite suggested by Chidamber and Kemerer (1994).

- Lack of Cohesion in Methods (LCOM3) suggested by Henderson-Sellers (1996).

- The QMOOD metrics suite suggested by Bansiya and Davis (2002).

- The quality oriented extension to Chidamber and Kemerer metrics suite suggested by Tang et al. (1999).

- Coupling metrics suggested by Martin (1994).

- Class level metrics built on the basis of McCabe's (1976) complexity metric.

- Lines of Code (LOC).

A separate report by Jureczko and Madeyski (2011c), available online, presents definitions of the aforementioned metrics.

\subsubsection{Process metrics}

A considerable research has been performed on identifying the process metrics which influence the efficiency of defect prediction. Among them, the most widely used are the metrics similar to NR, NDC, NML and NDPV (cf. Sect. 3): 
- Number of Revisions (NR). The NR metric constitutes the number of revisions (retrieved from a main line of development in a version control system, e.g., trunk in SVN) of a given Java class during development of the investigated release of a software system. The metric (although using different names) has already been used by several researchers (Graves et al. 2000; Illes-Seifert and Paech 2010; Moser et al. 2008; Nagappan and Ball 2007; Nagappan et al. 2010; Ostrand and Weyuker 2002; Ostrand et al. 2004; Ratzinger et al. 2007; Schröter et al. 2006; Shihab et al. 2010; Weyuker et al. 2006, 2007, 2008).

- Number of Distinct Committers (NDC). The NDC metric returns the number of distinct authors who committed their changes in a given Java class during the development of the investigated release of a software system. The metric has already been used or analyzed by researchers (Bell et al. 2006; Weyuker et al. 2007, 2008, 2010; Graves et al. 2000; Illes-Seifert and Paech 2010; Matsumoto et al. 2010; Moser et al. 2008; Nagappan et al. 2008, 2010; Ratzinger et al. 2007; Schröter et al. 2006; Zimmermann et al. 2009).

- Number of Modified Lines (NML). The NML metric calculates the sum of all lines of source code which were added or removed in a given Java class. Each of the committed revisions during the development of the investigated release of a software system is taken into account. According to the CVS version-control system, a modification in a given line of source code is equivalent to removing the old version and subsequently adding a new version of the line. Similar metrics have already been used or analyzed by various researchers (Graves et al. 2000; Hassan 2009; Purushothaman and Perry 2005; Layman et al. 2008; Moser et al. 2008; Nagappan and Ball 2005, 2007; Nagappan et al. 2008, 2010; Ratzinger et al. 2007; Śliwerski et al. 2005; Zimmermann et al. 2009).

- Number of Defects in Previous Version (NDPV). The NDPV metric returns the number of defects repaired in a given class during the development of the previous release of a software system. Similar metrics have already been investigated by a number of researchers (Arisholm and Briand 2006; Hassan 2009; Ostrand et al. 2005; Weyuker et al. 2006, 2008; Graves et al. 2000; Gyimothy et al. 2005; Illes-Seifert and Paech 2010; Kim et al. 2007; Khoshgoftaar et al. 1998; Moser et al. 2008; Nagappan et al. 2008, 2010; Ostrand and Weyuker 2002; Ratzinger et al. 2007; Schröter et al. 2006; Shihab et al. 2010; Śliwerski et al. 2005; Wahyudin et al. 2008).

\subsection{Tools}

All product metrics were calculated with the Ckjm tool ${ }^{2}$. The tool calculates all the aforementioned product metrics by processing the byte code of the compiled Java files.

The fact that the metrics are collected from byte code is not considered here as threat to the experiment, since-as it was explained in the case of LOC by Fenton and Neil (1999) - a metric calculated directly from the source code and the same metric calculated from the byte code are the alternative measures of the same attribute. The Ckjm version reported by Jureczko and Spinellis (2010) was used in this study.

The process metrics and the defect count were collected with a tool called BugInfo ${ }^{3}$. The BugInfo analyzes the logs from the source code repository (SVN or CVS) and, according to the log content, decides whether a commit is a bugfix. A commit is considered

\footnotetext{
2 http://gromit.iiar.pwr.wroc.pl/p_inf/ckjm/.

${ }^{3} \mathrm{http} / / /$ kenai.com/projects/buginfo.
} 
to be a bugfix when it solves an issue reported in a bug tracking system. Each of the projects was investigated in order to identify bugfixes commenting guidelines which had been used in the source code repository. The guidelines were formalized into regular expressions. Buginfo compares the regular expressions with the comments of the commits. When a comment matches the regular expression, BugInfo increments the defect count for all the classes which have been modified in the commit. The tool has been recently incorporated into a more complex one, i.e., QualitySpy (Jureczko and Magott 2012), which is under development. The QualitySpy tool was used to collect the NML metric from projects that use SVN repositories as such feature is not supported by BugInfo. Unfortunately, some time passed before the QualitySpy was ready to use, and hence, we faced obstacles (mostly significant changes in project structure that make impossible to match the newly collected data with the new ones) that prevented us from collecting the NML metric in some of the investigated projects.

Even though there is no formal evaluation of BugInfo regarding the efficiency in mapping defects yet, comprehensive tests have already been conducted. Most of them are available online as JUnit's tests in the BugInfo source code package.

\subsection{Analyzed projects}

Forty-three releases of 12 open source and 27 releases of 6 industrial software projects were investigated in this study.

In order to ensure consistent measurement of product metrics, all of the analyzed projects were written in Java. It is worth mentioning that we were not able to collect all of the process metrics for all of the projects. Therefore, some of the analyses were conducted on a subset of the projects mentioned below, hence each project has references to those experiments in which it was used, e.g., NR denotes that the project was analyzed in the experiment that investigated the NR metric. The experiment where all four metrics were investigated was executed on those projects that have references to all four metrics. The following projects were examined:

- POI version 1.5, 2.0, 2.5.1 and 3.0 (http://poi.apache.org, NR, NDC, NDPV).

- Synapse version 1.0, 1.1 and 1.2 (http://synapse.apache.org, NR, NDC, NML, NDPV).

- Xalan-Java version 2.4, 2.5, 2.6 and 2.7 (http://xml.apache.org/xalan-j, NR, NDC, NML, NDPV).

- Xerces version 1.1, 1.2, 1.3 and 1.4 .4 (http://xerces.apache.org/xerces-j, NR, NDC, NML, NDPV).

- Ant version 1.3, 1.4, 1.5, 1.6 and 1.7 (http://ant.apache.org, NR, NDC, NML, NDPV).

- PBeans version 1.0 and 2.0 (http://pbeans.sourceforge.net, only descriptive statistics).

- Ivy version 1.1, 1.2 and 2.0 (http://ant.apache.org/ivy, NR, NDC).

- Camel version 1.0, 1.2, 1.4 and 1.6 (http://camel.apache.org, NR, NDC, NML, NDPV).

- $\log 4 \mathrm{j}$ version 1.0, 1.1 and 1.2 (http://logging.apache.org/log4j, NR, NDC, NDPV).

- Lucene version 2.0, 2.2 and 2.4 (http://lucene.apache.org, NR, NDC, NML, NDPV).

- Velocity version 1.4, 1.5 and 1.6.1 (http://velocity.apache.org, NR, NDC, NDPV).

- JEdit version 3.2.1, 4.0, 4.1, 4.2 and 4.3 (http://www.jedit.org, NR, NDC, NML, NDPV).

- Five industrial projects belonging to the insurance domain (NR, NDC, NML, NDPV).

- One industrial project that is a tool that supports quality assurance in software development (NR, NDC). All six projects are developed by the same software development company, by international teams in a plan driven manner. However, only 
the insurance projects has fixed scope and deadline (for each release, respectively), whereas the sixth one has much more flexible plans. The five projects from the insurance domain are custom built solutions with more than five years of development history. They implement different feature sets according to the individual customer requirements. All of them are developed using Java enterprise technologies and frameworks, as well as already installed in customer environments.

A separate report by Jureczko and Madeyski (2011b), available online, presents the descriptions of the open-source projects for which the authors have built software defect prediction models. The collected data are available online in our Metric Repository ${ }^{4}$. Moreover, the repository contains also some metadata, e.g., the regular expressions used to identify defects. In order to obtain exactly the same data as used in this study, the following URL should be used: http://purl.org/MarianJureczko/MetricsRepo/ WhichMetricsImprovePrediction. Furthermore, the archive containing the same collection of data sets (called MJ12A) is available online ${ }^{5}$. It is worth mentioning that a number of analyses had already been conducted previously (Jureczko and Madeyski 2010; Jureczko and Spinellis 2010), but they were neither focused on process metrics nor did they use all of the data which we were able to collect and analyze in this paper (not to mention loosely related earlier study (Madeyski 2006) focused on external code quality instead of defects).

\section{Related work and background}

Comprehensive surveys on defect prediction were presented by Purao and Vaishnavi (2003), Catal and Diri (2009), Kitchenham (2010), and Hall et al. (2012). Hall et al. (2012) investigated how the context of prediction models (the modeling techniques applied and the independent variables used) affect the performance of fault prediction models. They synthesised the results of 36 studies and concluded that the methodology used to build models seems to be influential to predictive performance. For example, the models that perform comparatively well tend to be based on relatively simple modeling techniques (e.g., Naive Bayes, Logistic Regression), combinations of independent variables have been used by prediction models that perform well, while feature selection has been applied to these combinations when models are performing particularly well (Hall et al. 2012).

Moreover, considerable research has been performed on identifying the process metrics (similar to NR, NDC, NML and NDPV) which influence the efficiency of defect prediction. The research papers in which the aforementioned process metrics were employed or analyzed have been already mentioned in Sect. 2.1.2. This section describes a subset of these works which not only use but also are focused on analyses of the aforementioned process metrics. More comprehensive description is available in (Jureczko and Madeyski 2011a).

The NR metric (or a similar one) was recommended in all of the works presented in Table 1. However, it is worth mentioning that some of them reported only correlation coefficients with the number of defects (e.g., Illes-Seifert and Paech 2010; Schröter et al. 2006), while the other studies were carried out on limited data sets (i.e., single project): (Graves et al. 2000)—a telephone switching system, (Moser et al. 2008)—Eclipse, (Nagappan and Ball 2007)_Windows 2003 and (Nagappan et al. 2010)—Windows Vista.

\footnotetext{
${ }^{4}$ http://purl.org/MarianJureczko/MetricsRepo.

5 http://madeyski.e-informatyka.pl/tools/software-defect-prediction/.
} 
Table 1 Findings related to Number of Revisions

\begin{tabular}{ll}
\hline Who & Information and findings \\
\hline Graves et al. (2000) & $\begin{array}{c}\text { The change history contains more useful information than } \\
\text { could be obtained from the product (size and structure) } \\
\text { metrics } \\
\text { The number of lines of code of a module (a metric often used } \\
\text { in the defect prediction models) is not helpful in predicting } \\
\text { faults when the number of times a module was changed is } \\
\text { taken into account } \\
\text { In the case of pre-release failures, the number of changes had } \\
\text { the highest correlation coefficient among all the } \\
\text { investigated process and product metrics (.34-.47 in the } \\
\text { case of the Pearson's correlation and .44-.56 in the case of } \\
\text { the Spearman's correlation) } \\
\text { That research, performed on Eclipse, was later extended by } \\
\text { using the same metric in commercial projects (Nagappan } \\
\text { and Ball 2007) and by defining a metric which represents a } \\
\text { series of changes (Nagappan et al. 2010) } \\
\text { The process metric models and the combined (process and } \\
\text { product) models were more efficient than the product } \\
\text { metrics models }\end{array}$ \\
The Spearman's correlation of the Frequency of Change \\
metric with the number of defects was high in all nine \\
illes-Seifert and Paech (2010) \\
recommended as a very good defect indicator
\end{tabular}

Table 2 Findings related to Number of Distinct Committers

\begin{tabular}{|c|c|}
\hline Who & Information and findings \\
\hline Graves et al. (2000) & $\begin{array}{l}\text { A study of the code from a } 1.5 \text {-million-line subsystem of a telephone } \\
\text { switching system gave no evidence that a large number of developers } \\
\text { working on a module caused it to be more faulty }\end{array}$ \\
\hline Schröter et al. (2006) & $\begin{array}{l}\text { High correlation coefficient of the number of authors metric with pre- } \\
\text { and post-release failures }(.15-.41)\end{array}$ \\
\hline $\begin{array}{l}\text { Bell et al. (2006), and Weyuker } \\
\text { et al. }(2007,2008)\end{array}$ & $\begin{array}{l}\text { Adding developer's information to the defect prediction model resulted } \\
\text { in a slight improvement of the prediction efficiency }\end{array}$ \\
\hline $\begin{array}{l}\text { Matsumoto et al. (2010) and } \\
\text { Weyuker et al. (2010) }\end{array}$ & $\begin{array}{l}\text { Analysis of the relationship between a given developer and the density } \\
\text { of defects } \\
\text { Conflicting results with regard to usefulness of the approach }\end{array}$ \\
\hline Illes-Seifert and Paech (2010) & $\begin{array}{l}\text { High correlation coefficient of the number of commiters metric with } \\
\text { the number of defects(.16-.74) }\end{array}$ \\
\hline
\end{tabular}

Contradictory results related to the NDC metric were reported in the papers mentioned in Table 2. According to Weyuker et al. $(2007,2008)$, the metric improved the prediction performance, while as reported by Graves et al. (2000), the metric was not useful. Again, some researchers reported only the correlation coefficients(Illes-Seifert and Paech 2010; Schröter et al. 2006). Furthermore, Matsumoto et al. (2010) and Weyuker et al. (2010) recommended more sophisticated committer-related measurements.

Considerable research has been performed on the issue of the extent to which the NML of code affects the defect counts. The results presented in Table 3 are very encouraging 
Table 3 Findings related to Number of Modified Lines

\begin{tabular}{|c|c|}
\hline Who & Information and findings \\
\hline \multirow[t]{2}{*}{ Purushothaman and Perry (2005) } & Description of distribution of modification size \\
\hline & $\begin{array}{l}\text { Low probability of introducing an error in an one- } \\
\text { line change }(<4 \%)\end{array}$ \\
\hline Śliwerski et al. (2005) & $\begin{array}{l}\text { The larger the modification, the greater defect } \\
\text { introduction probability }\end{array}$ \\
\hline \multirow[t]{3}{*}{$\begin{array}{l}\text { Layman et al. (2008), Nagappan and Ball (2005) } \\
\text { and Nagappan et al. }(2008,2010)\end{array}$} & $\begin{array}{l}\text { Four different metrics related to the Number of } \\
\text { Modified Lines }\end{array}$ \\
\hline & $\begin{array}{l}\text { Defect prediction for Windows Server 2003, } \\
\text { Windows Vista }\end{array}$ \\
\hline & High prediction accuracy: $73.3-86.7 \%$ \\
\hline Hassan (2009) & $\begin{array}{l}\text { Module entropy (based on modification size) proved } \\
\text { to be useful in defect prediction }\end{array}$ \\
\hline
\end{tabular}

and suggest that there is a relation between the size of change and the likelihood of introducing defect. However, the investigated data sets were usually limited, the largest one (among the aforementioned) was investigated by Hassan (2009), i.e., five open-source projects. It should be stressed that there are also a number of metrics derived from the NML. Specifically, there are the studies in which the derived metrics are compared with the classical ones and show better performance with regard to defect prediction (e.g., Giger et al. 2011a, b). For the sake of simplicity, the classic version of NML was considered in this study. Nevertheless, it should not be ignored that there is a possibility that some of the derived metrics may perform better.

Another issue to which extensive research has been devoted is the extent to which the number of defects from the previous version impacts the defect counts in the current version. Most of the works reported in Table 4 suggest that the defects persists between the subsequent releases, however, there are also contrary results (Illes-Seifert and Paech 2010; Schröter et al. 2006). Furthermore, the scope of investigated projects could be considered unsatisfactory with regard to external validity. The greatest number of projects (i.e., 9) was investigated by Illes-Seifert and Paech (2010), but this study reported only the correlation coefficients and questioned the value of the NDPV metric.

\section{Study design}

This section presents the detailed description of the empirical investigation aimed at identifying the process metrics which may significantly improve simple defect prediction models based on product metrics.

\subsection{Statistical hypothesis}

In order to validate the usefulness of the process metrics in defect prediction, an empirical evaluation was conducted for each of the process metrics separately. The structure of the empirical evaluation process is described below. First, two kinds of models were constructed. The first model made use only of the product metrics and thus falls under the category of simple models and may be viewed as representative of the classic approach. 
Table 4 Findings related to Number of Defects in Previous Version

\begin{tabular}{|c|c|}
\hline Who & Information and findings \\
\hline $\begin{array}{l}\text { Khoshgoftaar et al. } \\
\text { (1998) }\end{array}$ & $\begin{array}{l}\text { The modules with faults in the past were claimed to be likely to have faults in the } \\
\text { future }\end{array}$ \\
\hline Graves et al. (2000) & $\begin{array}{l}\text { The model which predicted the number of faults as a constant multiple of the } \\
\text { number of faults that had been found in an earlier period of time showed to be } \\
\text { deficient but the authors took up the challenge of improving it }\end{array}$ \\
\hline $\begin{array}{l}\text { Ostrand and Weyuker } \\
\text { (2002) }\end{array}$ & $\begin{array}{l}\text { Moderate evidence that files remain high fault till later releases }(17-54 \% \text { of the } \\
\text { high-fault files of release } i \text { are still high fault in release } i+1)\end{array}$ \\
\hline Gyimothy et al. (2005) & $\begin{array}{l}\text { Correlations between the numbers of defects associated with the different versions } \\
\text { of classes (Mozilla versions } 1.0-1.6 \text { were analyzed) varied from } .69 \text { to } .9\end{array}$ \\
\hline Schröter et al. (2006) & $\begin{array}{l}\text { The correlation coefficients between pre- and post-release failures are smaller than } \\
\text { the correlation coefficients calculated from the two metrics mentioned before } \\
\text { (NR and NDC) }\end{array}$ \\
\hline \multirow[t]{2}{*}{ Kim et al. (2007) } & $\begin{array}{l}\text { A cache-based algorithm detected } 73-95 \% \text { of faults by selecting } 10 \% \text { of the } \\
\text { most fault-prone source code files }\end{array}$ \\
\hline & $\begin{array}{l}\text { Two of the principles behind the algorithm were connected with the NDPV } \\
\text { metric: temporal locality (If an entity introduces a fault recently, it will tend to } \\
\text { introduce other faults soon) and spatial locality (If an entity introduced a fault } \\
\text { recently, "nearby entities" (in the sense of logical coupling) will also tend to } \\
\text { introduce faults soon) }\end{array}$ \\
\hline $\begin{array}{l}\text { Illes-Seifert and Paech } \\
\text { (2010) }\end{array}$ & $\begin{array}{l}\text { "The number of defects found in the previous release of file does not correlate } \\
\text { with its current defect count." }\end{array}$ \\
\hline
\end{tabular}

The second model, which could be defined as an advanced model, used product metrics, as well as one process metric under investigation. Finally, the efficiency of prediction of the two types of models was compared. When the advanced models turned out to be significantly better than the simple ones, we calculated the effect size in order to assess whether the investigated process metric may be useful in practice of software defect prediction.

Let assume that: $r_{i}$ is the release number $i$ of a given project; $M r_{i}$ is a simple defect prediction model that was built on the release $r_{i}$ without using any process metric and $M^{\prime} r_{i}$ is an advanced defect prediction model that was built on the release $r_{i}$ with one process metric under investigation.

In order to create a simple model, all product metrics were used and the stepwise linear regression was applied. A typical model used five to ten metrics (but not all of them) depending on the selected method of stepwise regression.

In order to create a $M^{\prime}$ model, one of the process metrics was added to the set of the product metrics. Afterward, the same procedure was followed as the one described above for the simple model. It is also worth mentioning that we neither forced process metrics to be included in the advanced models, nor the advanced models always included the process metrics. Moreover, each of the advanced model that does not contain a process metric is exactly the same as its counterpart simple model.

Let assume that: $E\left(M r_{i}, r_{i+1}\right)$ is the evaluation of the efficiency of the model $M r_{i}$ in predicting defects in release $r_{i+1}$ and $E\left(M^{\prime} r_{i}, r_{i+1}\right)$ is the evaluation of the efficiency of the model $M^{\prime} r_{i}$ in predicting defects in release $r_{i+1}$. It should be stressed that the model built on release $i$ forms the basis for making predictions in release $i+1$ of the same project.

Let $n$ be the number of classes in release $r$. Let $c_{1}, c_{2}, \ldots, c_{n}$ denote the classes from release $r$ in descending order of numbers of predicted defects according to the model $M$, and $d_{1}, d_{2}, \ldots, d_{n}$ be the number of defects in each class. $D_{j}=\sum_{i=0}^{j} d_{i}$, i.e., the total 
defects in the first $j$ classes. Let $k$ be the smallest index so that $D_{k}>.8 \times D_{n}$, then $E(M, r)=k / n \times 100 \%$. The procedure has been expressed in a Visual Basic script which is available online http://purl.org/MarianJureczko/ProcessMetricsExperiments.

In order to decide whether the process metrics are useful in defect prediction, a statistical hypothesis was tested for each of the process metric separately:

- $H_{0, E\left(M^{\prime}\right)}$ - There is no difference in the efficiency of defect prediction between the simple model $(M)$ and the advanced model $\left(M^{\prime}\right)$.

Alternative hypothesis:

- $H_{1, E\left(M^{\prime}\right)}$ - There is a difference in the efficiency of defect prediction between the simple model $(M)$ and the advanced model $\left(M^{\prime}\right)$.

The hypotheses were evaluated by the parametric $t$ test for dependent samples. The homogeneity of variance was checked using Levene's test, while the assumption that the sample came from a normally distributed population was tested by way of the Shapiro-Wilk test (Madeyski 2010). If the aforementioned assumptions were violated, the nonparametric Wilcoxon matched pair test was used instead of its parametric counterpart, i.e., the dependent $t$ test. The investigated hypotheses were tested at the $\alpha=.05$ significance level.

\subsection{Effect size}

When the advanced models gave much better predictions, the effect size was calculated. Calculating the effect size estimations in the case of the dependent $t$ test was thoroughly discussed by Madeyski (2010). The crucial issue is that if an effect size is computed from the test statistics without taking into account the correlation between the repeated measures, the effect size will be overestimated (Dunlap et al. 1996; Madeyski 2010). The effect size calculation is based on the following procedure (Madeyski 2010):

$$
d=t_{r} \times \sqrt{\frac{2 \times\left(1-r_{r}\right)}{n}}
$$

where $r_{r}$ is the value of Pearson's correlation coefficient between the experimental and the control scores, $t_{r}$ is the repeated measures $t$ statistic, while $n$ is the sample size per group. Furthermore, the effect size $r$ can be calculated as follows (Madeyski 2010):

$$
r=\frac{d}{\sqrt{\left(d^{2}+4\right)}}
$$

This effect size estimation indicates the difference between the models according to the benchmark by Kampenes et al. (2007).

When nonparametric tests have been employed, an estimate of effect size $r$ has been obtained from the standard normal deviate $Z$ as suggested by Rosenthal (1991).

$$
r=\frac{Z}{\sqrt{N}}
$$

where $N$ is the number of sampling units on which $Z$ is based. However, in our opinion, the Rosenthal's approach could be called into question as it ignores the pairing effect. Hence, we also provide a nonparametric effect size measure referred to as probability of superiority (PS) recommended by Grissom and Kim (2012). They note that this measure can be 
extended to a dependent samples design involving ranked data, where PS is the proportion of cases in which a subject's score is higher in one experimental condition as opposed to the other, while ties are dropped from the analysis. We provide PS for the advanced models in case of nonparametric analyses.

Since we always used the previous version to train the model, there were no data to train a model that could be evaluated on the first version and there was no point in using the last version to train a model because there is no version which could serve to evaluate such a model. As a result, the number of evaluations is lower than the number of investigated project versions. Specifically, in the case of a project that consist of two versions, there is only one evaluation: The model trained on the first version is evaluated on the second one.

\section{Analysis}

In this section, we analyze the relationship between the number of defects in a software class and the values of process metrics.

\subsection{Descriptive statistics}

The descriptive statistics related to the process metrics, as well as the correlations between each of the process metrics and the numbers of defects, are presented below. As mentioned before, we were not able to collect all of the process metrics for all of the projects. As a result, if a particular process metric was not gathered for a project, the project was excluded from analysis. All statistics were calculated for the investigated projects both jointly (and denoted as "All projects"), as well as for each of the projects separately.

\subsubsection{Number of revisions (NR)}

The mean values of the NR metric (Table 5) are below 1 in all the industrial projects. That results from the fact that these projects are in the maintainability phase during which the developers work on new features, as well as fix defects, but the cores of these systems remain unchanged. The average number of class revisions was larger in the open-source projects. The smallest mean value for an open-source project is 1.88 , while the greatest value for an industrial projects equals .94 .

The correlation coefficients of the NR metric and the number of defects (Table 6) are high in most of the open-source projects (the Pearson' correlation coefficient typically is close to .5). In the case of the industrial projects, the correlations are smaller (typically close to .3). The value calculated for all the projects is dominated by the industrial projects, since those projects contain many more classes (the average number of classes in the industrial projects is 2580, whereas in the open-source projects it equals 350 ).

The Spearman's correlation is smaller than the Person correlation, and in two projects (Prop-5 and Poi) even falls below zero. Therefore, one may argue that the relation between the Number of Revisions metric and the defect count may be close to a linear one, and hence, it encourages to the further analysis where models with linear combination of metrics are used. The scatterplot (Fig. 1a) does not support that finding and shows a significant dispersion instead. However, the scatterplot presents all the investigated projects jointly, hence, if the linear relation is true only within projects (or versions), it can be not visible on this kind of chart. Therefore, the aforementioned analysis remains reasonable. 
Table 5 NR, NDC, NML and NDPV-descriptive statistics

\begin{tabular}{|c|c|c|c|c|c|c|c|c|}
\hline Project & $\begin{array}{l}\text { Mean } \\
(\mathrm{NR})\end{array}$ & $\begin{array}{l}\text { SD } \\
(\mathrm{NR})\end{array}$ & $\begin{array}{l}\text { Mean } \\
(\text { NDC) }\end{array}$ & $\begin{array}{l}\text { SD } \\
\text { (NDC) }\end{array}$ & $\begin{array}{l}\text { Mean } \\
(\text { NML) }\end{array}$ & $\begin{array}{l}\text { SD } \\
(\mathrm{NML})\end{array}$ & $\begin{array}{l}\text { Mean } \\
\text { (NDPV) }\end{array}$ & $\begin{array}{l}\text { SD } \\
(\mathrm{NDPV})\end{array}$ \\
\hline All projects & 1.27 & 3.54 & .68 & 1.19 & 19.77 & 113.65 & .26 & 1.02 \\
\hline Prop-1 & .78 & 2.28 & .52 & 1.19 & 26.01 & 151.46 & .32 & 1.20 \\
\hline Prop-2 & .94 & 2.22 & .59 & 1.11 & 20.63 & 104.33 & .19 & .80 \\
\hline Prop-3 & .51 & 1.40 & .39 & .82 & 14.73 & 90.53 & .14 & .47 \\
\hline Prop-4 & .41 & 1.37 & .31 & .83 & 10.95 & 80.27 & .11 & .53 \\
\hline Prop-5 & .47 & 1.17 & .37 & .75 & 18.07 & 83.75 & .27 & .76 \\
\hline Prop-6 & .56 & .99 & .41 & .61 & & & & \\
\hline Xalan & 1.98 & 1.86 & 1.25 & .87 & 104.03 & 337.58 & .49 & .88 \\
\hline Xerces & 1.88 & 3.89 & 1.00 & .98 & 179.56 & 640.59 & .24 & .81 \\
\hline Ant & 7.82 & 7.14 & 3.40 & 1.72 & 141.98 & 265.82 & .19 & .67 \\
\hline Ivy & 6.70 & 9.30 & 1.41 & .72 & & & .95 & 3.25 \\
\hline Camel & 1.95 & 3.46 & 1.03 & 1.24 & 27.42 & 93.51 & .35 & 1.25 \\
\hline $\log 4 j$ & 3.22 & 5.10 & 1.14 & .87 & & & .44 & 1.17 \\
\hline Lucene & 2.73 & 4.72 & 1.52 & 1.18 & 85.73 & 443.79 & 1.16 & 2.92 \\
\hline Poi & 2.90 & 2.98 & 1.90 & 1.10 & & & .76 & 1.51 \\
\hline Synapse & 3.17 & 3.15 & 1.57 & 1.05 & 95.16 & 155.96 & .25 & .73 \\
\hline Velocity & 5.12 & 9.74 & 1.47 & 1.21 & & & .97 & 1.90 \\
\hline JEdit & 6.51 & 12.46 & 1.54 & 1.55 & 126.63 & 583.98 & .63 & 2.34 \\
\hline Pbeans & & & & & & & .57 & 1.06 \\
\hline
\end{tabular}

\subsubsection{Number of distinct committers (NDC)}

The mean values of the NDC metric (Table 5) reflect the maintainability phase of the industrial projects. The mean value of the NDC metric is smaller in the case of the industrial projects. The Pearson's and Spearman's correlations between the number of defects and the metrics (given in Table 6) show a noticeable difference between opensource and industrial projects. The correlation coefficients are slightly smaller than in the case of the NR metric. The relation between the number of defects and the NDC metric is detailed in Fig. 1b. It supports the aforementioned conclusions about smaller correlation by showing large dispersion.

\subsubsection{Number of modified lines (NML)}

Number of Modified Lines metric values were collected from the five industrial projects using the BugInfo tool, as those projects used CVS as version control system. Later on the QualitySpy tool was used to mine the metric from the SubVersion repositories of 7 opensource projects.

Most of the Pearson's and Spearman's correlations between the process metrics and the number of defects (Table 6) are close to .3. The correlation between NML and the number of defects is similar as in the case of the NDC metrics. Nevertheless, the relation between the number of defects and the NML metric presented in Fig. 1c does not indicate linear relation. 
Table 6 NR, NDC, NML and NDPV-correlations with the number of defects

\begin{tabular}{|c|c|c|c|c|c|c|c|c|}
\hline Project & $\begin{array}{l}\text { Pear. } \\
\text { cor. } \\
\text { (NR) }\end{array}$ & $\begin{array}{l}\text { Spear. } \\
\text { cor. } \\
\text { (NR) }\end{array}$ & $\begin{array}{l}\text { Pear. } \\
\text { cor. } \\
\text { (NDC) }\end{array}$ & $\begin{array}{l}\text { Spear. } \\
\text { cor. } \\
\text { (NDC) }\end{array}$ & $\begin{array}{l}\text { Pear. cor. } \\
\text { (NML) }\end{array}$ & $\begin{array}{l}\text { Spear. } \\
\text { cor. } \\
\text { (NML) }\end{array}$ & $\begin{array}{l}\text { Pear. } \\
\text { cor. (NDPV) }\end{array}$ & $\begin{array}{l}\text { Spear. cor. } \\
\text { (NDPV) }\end{array}$ \\
\hline All projects & $.31 *$ & $.28 *$ & $.27 *$ & $.27 *$ & $.27^{*}$ & $.20 *$ & $.36^{*}$ & $.24 *$ \\
\hline Prop-1 & $.32 *$ & $.31 *$ & $.33 *$ & $.31 *$ & $.25^{*}$ & $.31 *$ & $.35^{*}$ & $.32 *$ \\
\hline Prop-2 & $.30 *$ & $.16^{*}$ & $.25^{*}$ & $.16^{*}$ & $.26^{*}$ & $.17^{*}$ & $.35^{*}$ & $.22 *$ \\
\hline Prop-3 & $.38 *$ & $.12 *$ & $.28 *$ & $.12 *$ & $.32 *$ & $.13^{*}$ & $.11^{*}$ & $.03 *$ \\
\hline Prop-4 & $.49^{*}$ & $.40 *$ & $.34 *$ & $.40 *$ & $.36^{*}$ & $.38 *$ & $.30 *$ & $.15^{*}$ \\
\hline Prop-5 & $.27 *$ & -.02 & $.13 *$ & -.03 & $.24 *$ & -.01 & $.24 *$ & $-.05^{*}$ \\
\hline Prop-6 & $.16^{*}$ & $.14^{*}$ & $.12 *$ & $.13^{*}$ & & & & \\
\hline Xalan & $.35^{*}$ & $.10 *$ & $.20 *$ & $.12 *$ & .14 & .18 & $.40^{*}$ & $.31 *$ \\
\hline Xerces & $.24 *$ & $.09 *$ & $.29 *$ & $.11 *$ & .36 & .29 & $.11^{*}$ & $.10^{*}$ \\
\hline Ant & $.54 *$ & $.37 *$ & $.36^{*}$ & $.30 *$ & .46 & .42 & $.43^{*}$ & $.29 *$ \\
\hline Ivy & $.50 *$ & $.35^{*}$ & $.32 *$ & $.26^{*}$ & & & $.55^{*}$ & $.19 *$ \\
\hline Camel & $.54 *$ & $.52 *$ & $.41 *$ & $.49 *$ & .52 & .57 & $.41 *$ & $.32 *$ \\
\hline $\log 4 j$ & $.49 *$ & $.51 *$ & $.50 *$ & $.50 *$ & & & $.38 *$ & $.23 *$ \\
\hline Lucene & $.67 *$ & $.34 *$ & $.45^{*}$ & $.31 *$ & .53 & .54 & $.67 *$ & $.34 *$ \\
\hline Poi & $.19 *$ & -.03 & $.22 *$ & .02 & & & $.26^{*}$ & $.09 *$ \\
\hline Synapse & $.40 *$ & $.27 *$ & $.26^{*}$ & $.23 *$ & .26 & .26 & $.37 *$ & $.24^{*}$ \\
\hline Velocity & $.42 *$ & $.27 *$ & $.26 *$ & $.15^{*}$ & & & $.52 *$ & $.46^{*}$ \\
\hline JEdit & $.56^{*}$ & $.36 *$ & $-.08^{*}$ & $-.10^{*}$ & .57 & .41 & $.74 *$ & $.45^{*}$ \\
\hline Pbeans & & & & & & & $.63 *$ & $.39 *$ \\
\hline
\end{tabular}

* Correlations significant at .05 level

\subsubsection{Number of defects in previous version (NDPV)}

The great diversity in the correlations with the number of defects (Table 6) among projects may be illustrated by the fact that there is a project with the correlation equal .74-but also another one, with the correlation value close to zero.

That suggests that the Number of Defects in Previous Version may be very useful in defect prediction in some projects, but not in all of them. Fortunately, Fig. 1d shows large dispersion between the NDPV metric and the number of defects when all the projects are analyzed jointly.

\subsubsection{Relationships between metrics}

The process metrics are expected to improve the prediction performance. The improvement is possible when the metrics constitute an extra source of information, the information that is not available in the product metrics. The aforementioned condition is not satisfied when the process metrics are strongly correlated with the product metrics. Moreover, there are several different process metrics taken into consideration. Correlations between these metrics are relevant as well, since there is no point in adding two different metrics highly correlated with each other. The correlation analysis is presented in Table 7. 
The Pearson's correlations suggest that the process metrics constitute a different source of information. The greatest correlation coefficient between process and product metric was obtained in the case of the NR and RFC metrics and was equal to .39. Nevertheless, most of the coefficients are below .2 and hence can be considered as low. There is a different situation in the case of the correlations between process metrics, since the coefficients vary from .41 to .71. In consequence, it is questionable whether these metrics describe different attributes and whether adding more than one of them will significantly improve prediction performance.

\subsection{Hypotheses testing}

The analysis of each of the aforementioned process metrics is presented in the subsequent subsections. However, it is worth mentioning that it was not possible to collect all of the process metrics for all of the projects and versions. The simple models were always constructed using only the versions of the projects for which the process metrics investigated at that time were available. Therefore, different assessment results were obtained for the simple models in different experiments. The advanced models were constructed separately for each version of each project and each process metric (see Sect. 4 for details). Additional details about the experiments (in particular all the simple and advanced models) are available online: http://purl.org/MarianJureczko/ProcessMetricsExperiments.

\subsubsection{Number of revisions (NR)}

Thirty-four versions of projects were analyzed. According to Table 8, on average $56.86 \%$ of classes must be tested in order to find $80 \%$ of defects when the simple model is used, and only $53.83 \%$ of classes when the advanced model is used. However, according to Table 9 , the difference is not statistically significant at the $\alpha=.05$ significance level. Therefore, the $H_{0, E\left(M^{\prime}\right)}$ hypothesis may not be rejected in this case. Even though the NR metric did not prove to be radically useful in building defect prediction models, the advanced models gave slightly better predictions. That suggests that the metric perhaps should not be entirely left out in the process of defect prediction model construction.

On the basis of the effect size calculation procedure presented in Sect. 4 and collected data $\left(r_{r}=.846\right.$ is the value of Pearson's correlation coefficient between the experimental and the control scores, $t_{r}=1.485$ is the repeated measures $t$ statistic, and $n=34$ is the sample size per group), we calculated the effect size measure $r=.07$. This effect size estimation indicates that the difference between the models represents a small effect according to the benchmark by Kampenes et al. (2007).

Our conclusion is that the $H_{0, E\left(M^{\prime}\right)}$ hypothesis may not be rejected in this case, as the efficiency of the simple and the advanced models (trained on a data set containing product metrics and additionally the NR metric) did not significantly differ $(t(33)=1.48$, $p=.147, r=.07)$.

\subsubsection{Number of distinct committers (NDC)}

Thirty-four versions of projects were analyzed. According to Table 10, the advanced models outperform the simple ones. Moreover, according to Table 11, the difference in prediction efficiency is statistically significant $(p=.015)$. Wilcoxon Matched Pair Test was employed since according to the Shapiro-Wilk test the normality assumption was 

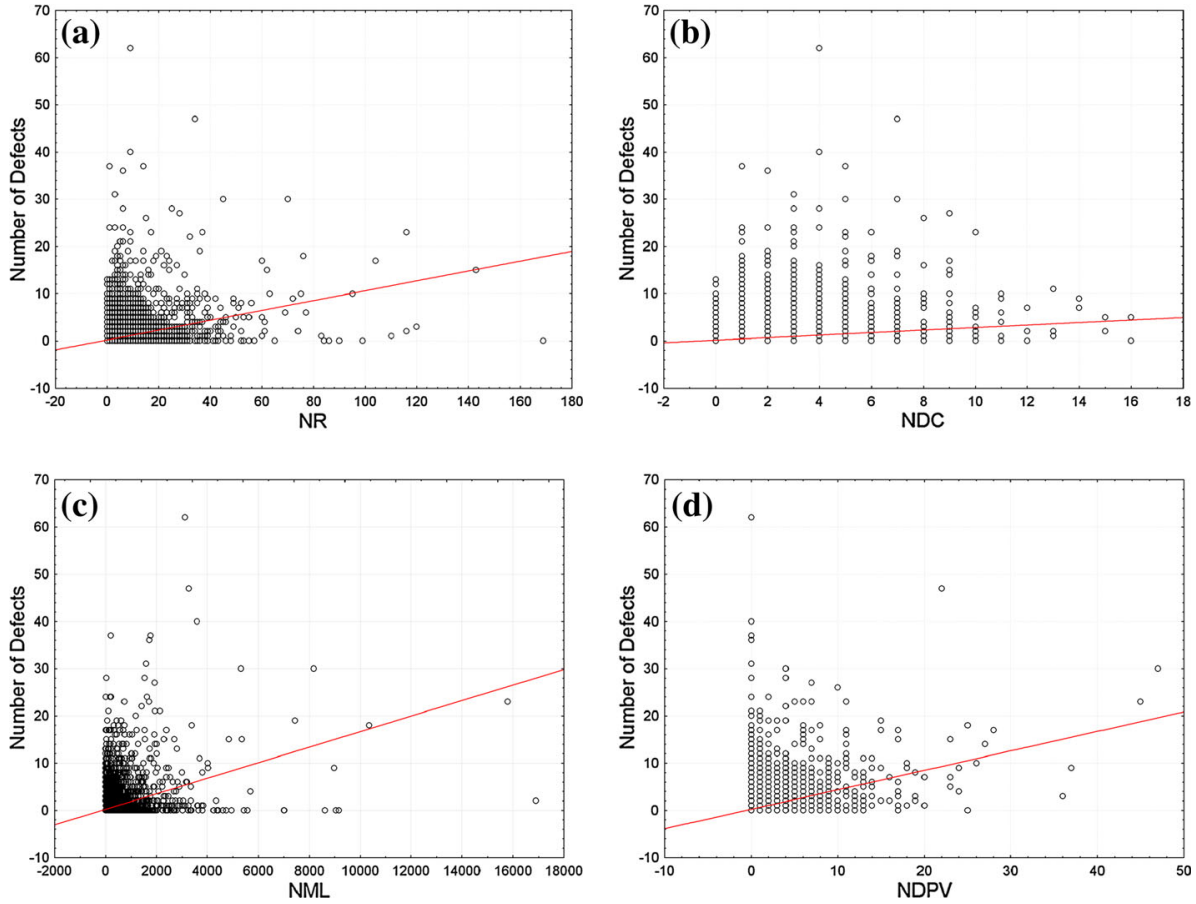

Fig. 1 Scatterplots of number of defects against process metrics. a NR. b NDC. c NML. d NDPV

violated. Therefore, the $H_{0, E\left(M^{\prime}\right)}$ hypothesis may be rejected in this case and we may conclude that the NDC metric is useful in building defect prediction models.

On the basis of the effect size calculation procedure presented in Sect. 4, we calculated the PS for the advanced models PS $=.76$ and the effect size measure $r=-.29$. This effect size estimation indicates that the difference between the models represents a medium effect according to the benchmark by Kampenes et al. (2007). It denotes that the result of using the NDC metric in prediction models created a medium effect in the efficiency of the models $(p=.016, r=-.29$, PS $=.76$ ), which is a substantial finding, useful in defect prediction.

\subsubsection{Number of modified lines (NML)}

Twenty-seven versions of projects were analyzed. According to Table 12, the advanced model is more efficient than the simple model. The Table 13 points to the fact that the difference is statistically significant $(p=.038)$. Therefore, we may conclude that the NML metric improves the efficiency of the defect prediction models-the $H_{0, E\left(M^{\prime}\right)}$ hypothesis may be rejected.

On the basis of the effect size calculation procedure presented in Sect. 4, we calculated the effect size measure $r=.06$. This effect size estimation indicates that the difference between the models represents a small effect according to the benchmark by Kampenes et al. (2007). Hence, our conclusion is that while the efficiency of the simple and the 
Table 7 Pearson's correlations

\begin{tabular}{|c|c|c|c|c|}
\hline & NR & NDC & NML & NDPV \\
\hline NR & 1.00 & .71 & .60 & .53 \\
\hline NDC & .71 & 1.00 & .37 & .43 \\
\hline NML & .61 & .49 & 1.00 & .41 \\
\hline NDPV & .53 & .43 & .36 & 1.00 \\
\hline WMC & .33 & .19 & .36 & .25 \\
\hline DIT & -.08 & -.11 & -.04 & -.05 \\
\hline NOC & .01 & .01 & .00 & .01 \\
\hline $\mathrm{CBO}$ & .23 & .20 & .14 & .25 \\
\hline RFC & .39 & .28 & .36 & .35 \\
\hline LCOM & .15 & .04 & .16 & .12 \\
\hline $\mathrm{CA}$ & .21 & .16 & .10 & .17 \\
\hline $\mathrm{CE}$ & .14 & .13 & .13 & .23 \\
\hline NPM & .27 & .16 & .25 & .21 \\
\hline LCOM3 & -.09 & -.07 & -.06 & -.07 \\
\hline LOC & .33 & .24 & .48 & .30 \\
\hline DAM & .16 & .14 & .10 & .06 \\
\hline MOA & .22 & .13 & .18 & .13 \\
\hline MFA & -.08 & -.09 & -.05 & -.05 \\
\hline CAM & -.22 & -.23 & -.15 & -.16 \\
\hline IC & -.05 & -.08 & -.03 & -.02 \\
\hline CBM & .02 & -.02 & -.03 & .02 \\
\hline AMC & .08 & .12 & .20 & .10 \\
\hline $\operatorname{Max}(\mathrm{CC})$ & .22 & .19 & .22 & .19 \\
\hline $\operatorname{Avg}(C C)$ & .13 & .14 & .12 & .13 \\
\hline
\end{tabular}

Table 8 Number of revisionsevaluation results

\begin{tabular}{llll}
\hline & Mean & Median & SD \\
\hline$E(M)$ & 56.86 & 59.37 & 17.58 \\
$E\left(M^{\prime}\right)$ & 53.83 & 53.90 & 22.15 \\
\hline
\end{tabular}

advanced models (trained on a data set containing product metrics and additionally the NML metric) differ significantly, the effect size is small $(t(26)=2.19, p=.038, r=.06)$.

\subsubsection{Number of defects in previous version (NDPV)}

Thirty-three versions of projects were analyzed. There is only a small difference between the efficiency of the simple and the advanced model (Table 14). According to Table 15, the difference is not statistically significant $(p=.953)$. Therefore, there is no basis for rejecting the $H_{0, E\left(M^{\prime}\right)}$ hypothesis.

We calculated the effect size measure $r=-.003$ on the basis of the procedure presented in Sect. 4. This effect size estimation points that the difference between models represents a small effect according to the benchmark by Kampenes et al. (2007). Hence, 
Table 9 Number of revisionshypothesis test

\begin{tabular}{llll}
\hline & & $E(M)$ & $E\left(M^{\prime}\right)$ \\
\hline Shapiro-Wilk test & $\mathrm{W}$ & .97 & .94 \\
& $\mathrm{p}$ & .39 & .07 \\
\multirow{2}{*}{$t$ test } & $\mathrm{t}$ & 1.48 & \\
& $\mathrm{df}$ & 33 & \\
& $\mathrm{p}$ & .147 & \\
\hline
\end{tabular}

\begin{tabular}{llll}
\hline & Mean & Median & SD \\
\hline$E(M)$ & 56.86 & 59.37 & 17.58 \\
$E\left(M^{\prime}\right)$ & 52.88 & 56.98 & 20.08 \\
\hline
\end{tabular}

Table 10 Number of Distinct Committers-evaluation results

Table 11 Number of Distinct Committers-hypothesis test

Bold value is statistically significant

\begin{tabular}{llll}
\hline & & $E(M)$ & $E\left(M^{\prime}\right)$ \\
\hline Shapiro-Wilk test & $\mathrm{W}$ & .97 & .93 \\
& $\mathrm{p}$ & .39 & .04 \\
Wilcoxon matched & $\mathrm{Z}$ & 2.44 & \\
Pair test (2-tailed) & $\mathrm{p}$ & $\mathbf{. 0 1 6}$ & \\
\hline
\end{tabular}

the efficiency of the simple and the advanced models (trained on a data set containing product metrics and additionally the NDPV metric) did not significantly differ and the effect size is tiny $(t(33)=-.06, p=.953, r=-.003)$.

\subsubsection{Combination of process metrics}

Four different process metrics were introduced at the same time to the defect prediction models in the last experiment. The models were built using all the product metrics and four process metrics: NR, NDC, NML and NDPV.

Table 16 shows that the advanced models give slightly better predictions. The mean values and medians of the efficiency of the simple and the advanced models do not lead to clear conclusions. That is not surprising, as-according to Table 17-the difference was not statistically significant.

On the basis of the effect size calculation procedure presented in Sect. 4, we derived the PS for the advanced models PS $=.68$ and the effect size measure $r=-.29$. This effect size estimation indicates that the difference between models represents a medium effect according to the benchmark by Kampenes et al. (2007). In conclusion, the efficiency of the simple model (including only the product metrics) and the models trained on a data set containing both product and process metrics differ significantly $(p=.038, r=-.29$, $\mathrm{PS}=.68$ ). It is worth mentioning that similar or even slightly better results (taking into account the PS of the advanced models) were obtained in the case of advanced models that employed only the NDC metric and it is not questionable that collecting one process metric requires less effort than collecting four of them. 
Table 12 Number of Modified Lines-evaluation results

\begin{tabular}{llll}
\hline & Mean & Median & SD \\
\hline$E(M)$ & 52.07 & 54.59 & 15.54 \\
$E\left(M^{\prime}\right)$ & 50.21 & 52.23 & 15.77 \\
\hline
\end{tabular}

\begin{tabular}{lllll} 
Table 13 Number of Modified & & & $E(M)$ & $E\left(M^{\prime}\right)$ \\
\cline { 2 - 4 } \begin{tabular}{l} 
Lines-hypothesis test \\
\cline { 2 - 4 }
\end{tabular} & Shapiro-Wilk test & $\mathrm{W}$ & .93 & .93 \\
& & $\mathrm{p}$ & .07 & .08 \\
& & $\mathrm{t}$ & 2.19 & 26 \\
Best & & $\mathrm{df}$ & $\mathbf{0 3 8}$ \\
Bold value is statistically & & $\mathrm{p}$ &
\end{tabular}

Table 13 Number of Modified Lines-hypothesis test

Bold value is statistically significant

\subsection{Association and subgroup analyses}

To see whether there are some patterns in open-source and industrial projects in the context of process metrics analyzed in the paper, we performed additional association and subgroup analyses.

For each of the process metric analyzed in this paper, we conducted Pearson's $\chi^{2}$ test to examine the association between two categorical variables, i.e., whether a project comes from an industrial environment or not (in our case it means an open-source environment), and whether the advanced model outperformed the simple model for a particular software project and process metric. These simple analyses produced the values of the $\chi^{2}$ statistic which are not significant. Hence, we are not able to recommend a specific process metric to improve defect prediction models in a particular environment (industrial or open source).

Subgroup analysis reveals that in 8 of 19 releases of open-source projects, none of the process metrics improved prediction models (i.e., in 8 releases none of the advanced models was better than simple models). However, in all of the releases of industrial projects at least one process metric improved prediction models, i.e., it was possible to build an advanced model which was better than simple model for each of the industrial project release and at least one process metric.

\section{Threats to validity}

The usefulness of empirical results depends on their importance and validity. The importance has been stressed in Sect. 1 and demonstrated by a number of publications mentioned in Sect. 3.

The validity of our results is discussed in this section on the basis of a comprehensive classification of threats to validity given by Cook and Campbell (1979), later refined by Shadish et al. (2002) and used within the empirical software engineering domain (Wohlin et al. 2000; Madeyski 2010). This classification consists of four related components: construct validity, statistical conclusion validity, internal validity and external validity. 
Table 14 Number of Defects in Previous Version-evaluation results

Table 15 Number of Defects in Previous Version-hypothesis test

Table 16 Combination of process metrics-evaluation results

Table 17 Combination of process metrics-hypothesis test

Bold value is statistically significant

\begin{tabular}{llll}
\hline & Mean & Median & SD \\
\hline$E(M)$ & 55.77 & 58.27 & 17.35 \\
$E\left(M^{\prime}\right)$ & 55.87 & 61.82 & 19.12 \\
\hline
\end{tabular}

\begin{tabular}{llll}
\hline & & $E(M)$ & $E\left(M^{\prime}\right)$ \\
\hline Shapiro-Wilk test & $\mathrm{W}$ & .97 & .95 \\
& $\mathrm{p}$ & .55 & .14 \\
\multirow{3}{*}{$t$ test } & $\mathrm{t}$ & -.06 & \\
& $\mathrm{df}$ & 32 & \\
& $\mathrm{p}$ & .953 & \\
\hline
\end{tabular}

\begin{tabular}{llll}
\hline & Mean & Median & SD \\
\hline$E(M)$ & 52.69 & 56.44 & 15.52 \\
$E\left(M^{\prime}\right)$ & 49.06 & 52.54 & 20.26 \\
\hline
\end{tabular}

\begin{tabular}{llll}
\hline & & $E(M)$ & $E\left(M^{\prime}\right)$ \\
\hline Shapiro-Wilk test & $\mathrm{W}$ & .92 & .06 \\
& $\mathrm{p}$ & .92 & .04 \\
Wilcoxon & $\mathrm{T}$ & 62 & \\
Matched & $\mathrm{Z}$ & -2.08 & \\
Pair test & $\mathrm{p}$ & $\mathbf{. 0 3 8}$ & \\
\hline
\end{tabular}

\subsection{Construct validity}

Threats to construct validity concern the extent to which inferences can legitimately be made from the operationalizations in our study to the theoretical constructs on which those operationalizations were based. The mono-method bias regards a single means of recording measures. When all operationalizations use the same method, the method becomes a part of the construct. Hence, an important construct validity threat is that it was not possible to assure that all the links between bugs and versioning system files (i.e., classes) are retrieved (e.g., when the bug reference identifier is missing in the commit comment, as bugs are identified according to the content of the comments). This problem is common and so far unresolved in the area of linking bugs to versioning system files and classes, and the method we implemented in this study is both widely used and state of the art in addressing the issue (Bacchelli et al. 2010; D'Ambros et al. 2010a, b; Fischer et al. 2003; Zimmermann et al. 2007). However, if a more reliable linking method became available, it would help to reduce that threat.

Inner classes are connected with another threat to the construct validity. It is not possible to decide whether a bug is related to inner classes or their containing class since the source code version control systems have a file-based nature. Java inner classes are defined 
in the same file as their containing class. Therefore, not taking inner classes into consideration has become a common practice (Bacchelli et al. 2010; D’ Ambros et al. 2010a, b). What seems to be the advantage is that the proportion of inner classes in all classes is very small (in our experiment it is $8.84 \%$ ).

Different bugfix commenting guidelines are used in different software projects. Furthermore, the definition of a bug is not uniform among projects. According to Antoniol et al. (2008), a fraction of issues marked as bugs are problems unrelated to corrective maintenance. Such occurrences were removed manually.

We were not able to track the changes in a class name or moving a class between the packages. Unfortunately, usually after such a change the class had to be interpreted as a new one.

\subsection{Statistical conclusion validity}

Threats to statistical conclusion validity are related to the issues that affect the validity of inferences. Our study adopted robust statistical tools SPSS and Statistica as a double check. However, it is worth mentioning that calculation of effect size estimation for nonparametric tests, although suggested by Rosenthal (1991), ignores the pairing effect which could be called into question. Hence, nonparametric effect size estimations were calculated as well.

The process metrics were evaluated in this study, using a particular method of building the defect prediction model, namely stepwise linear regression. However, it would be interesting to replicate the study using different methods, e.g., neural networks.

\subsection{Internal validity}

The threats to internal validity refer to the misinterpretation of the true causes which affect the experiment result. According to D'Ambros et al. (2010a), human factor should be considered as an external factor which may affect the internal validity. The relevant human factor in defect prediction studies is bug severity. Since, according to Ostrand et al. (2004), the severity ratings are highly subjective and inaccurate, we decided to exclude them from the study.

It should be mentioned that the NR metric, if not precisely defined, can be misleading based on the way of working or commit habits of developers in version control systems. For instance, developers may not commit as frequent as they should due to concerns about breaking builds or incomplete work. Developers may also work in branches which may not be tracked by the measurement infrastructure. This might also explain the low numbers in the NR metric. However, we decided to use the metric for several reasons. First, we have emphasized that the NR metric we use should be understood as the number of revisions (retrieved from a main line of development in a version control system, e.g., trunk in SVN) of a given Java class during development of the investigated release of a software system. Second, in case of industrial projects, we only had access to specific main lines of development in a limited time frame. Third, this metric (although using different names) has also been used by other researchers without mentioning issues related to commit habits of developers, e.g. (Graves et al. 2000; Illes-Seifert and Paech 2010; Moser et al. 2008; Nagappan and Ball 2007; Nagappan et al. 2010; Ostrand and Weyuker 2002; Ostrand et al. 2004; Ratzinger et al. 2007; Schröter et al. 2006; Shihab et al. 2010; Weyuker et al. 2006, 2007, 2008), so we decided not to exclude the metric up front. The more so that the metric is relatively easy (cheap) to collect which is important in industrial environments. 
Table 18 Metrics evaluations

\begin{tabular}{llll}
\hline Metric & $\begin{array}{l}\text { Prediction } \\
\text { efficiency }\end{array}$ & $\begin{array}{l}\text { Statistically } \\
\text { significant }\end{array}$ & $\begin{array}{l}\text { Effect } \\
\text { size }\end{array}$ \\
\hline $\begin{array}{l}\text { Number of Revisions (NR) } \\
\begin{array}{l}\text { Number of Distinct } \\
\text { Committers (NDC) }\end{array}\end{array}$ & Better & No & Small \\
$\begin{array}{l}\text { Number of Modified Lines } \\
\text { (NML) }\end{array}$ & Better & Yes & Medium \\
$\begin{array}{l}\text { Number of Defects in } \\
\text { Previous Version (NDPV) }\end{array}$ & Not better & No & Small \\
$\begin{array}{c}\text { Combination of process } \\
\text { metrics }\end{array}$ & Better & Yes & Medium \\
\hline
\end{tabular}

\subsection{External validity}

External validity is related to generalizing the results of our study to other settings (usually industrial setting) or population.

This study investigates a wide range of different kinds of software projects. It covers two different source code ownership models (industrial and open source), different domains and different software development processes. Nevertheless, we only examined the projects written in Java. However, due to this limitation, all the code metrics are defined identically for each system, and therefore, the parsing bias was alleviated. The investigated projects are by no means representative and that poses a threat to the external validity, since there are studies (Zimmermann et al. 2009; Jureczko and Madeyski 2010) which show that the differences between software projects are sometimes large (at least from the point of view of defect prediction).

\section{Discussion of results}

The summary of our results is presented in Table 18 .

The NDC and NML metrics were valuable with regard to defect prediction. Both metrics significantly improved the efficiency of prediction and, therefore, are worth taking into account while building the defect prediction models. The effect size estimations were calculated for each of those metrics and subsequently compared against the benchmark by Kampenes et al. (2007). Small effects were obtained in the case of NML, while a medium effect in the case of NDC. Therefore, the usefulness of the NDC metric should not be doubted, but the NML metrics need further investigation.

In the case of the NR and NDPV metrics, no statistically significant results were obtained. The NR metric improved the model, but the difference was small. The NDPV metric only slightly affected the model.

Defect prediction models which utilize four different process metrics (NR, NDC, NML and NDPV) were investigated as well. The models usually gave better predictions than the models which did not use the process metrics at all and the difference was statistically significant. The prediction improvement was similar as in the case of the NDC metric. ${ }^{6}$ This finding suggests that the process metrics should be chosen carefully instead of

\footnotetext{
${ }^{6}$ Please note that different data set was used when investigating NDC, and hence more appropriate could be a comparison with the NML metric.
} 
applying all the available ones. In the investigated cases, introducing only one process metric (i.e., NDC) at a time resulted in similar or even slightly better models than the introduction of four process metrics simultaneously. The result can be explained to some extent by the correlation analysis, which showed that the process metrics are correlated with each other.

Association analyses described in Sect. 5.3 do not allow us to recommend a specific process metric to improve defect prediction models in a particular environment (industrial or open source). However, simple subgroup analysis revealed that in the industrial environment process metrics helped to improve simple prediction models for each of the industrial project release and at least one process metric, while in some of releases of opensource projects none of the process metrics improved prediction models. A possible explanation would be inherent differences between industrial and open-source environments.

\section{Conclusions and contributions}

Four different process metrics were analyzed in order to evaluate their usefulness in the defect prediction models. Moreover, the number of the investigated projects was large enough to obtain statistically significant results.

There is an interesting relation between the correlation of a metric with the number of defects and the usefulness of a metric in defect prediction models. The highest correlations were obtained in the case of the NR and NDPV metrics, while the most useful metrics with regard to the defect prediction models were NDC and NML. It can be seen as an example which confirms standard knowledge in Machine Learning that it is not enough to investigate a correlation exclusively. A high correlation coefficient with the number of defects does not necessary indicate that the metric will be useful in a defect prediction model. For the purpose of providing a better explanation of that relation, it would be necessary to perform the multicollinearity analysis.

The obtained results lead to the conclusion that the process metrics constitute a different source of information than the product metrics. Especially interesting are the NDC and NML metrics. The NDC metric regards the number of different developers who have changed a given class. When several people are involved in the process of development, it is always probable that they will misunderstand one another's intentions and overwrite the modifications made by their colleagues, which may, in turn, result in defects. It does not correspond with the shared code rule, popularized by the agile software development, by showing that such code ownership model is not optimal with regard to defects. Nevertheless, the shared code rule has also other consequences, e.g., impact on the learning curve or productivity. The NML metric reflects the size of change and according to the obtained results, the more lines of code are affected, the greater number of defects to be expected. The early approaches to defect prediction (from '70) postulated a linear relation between the lines of code (LOC) and the number of defects. The result obtained in the case of the NML metric partly moves this rule to the world of process metrics. There is a relation between the size of code and defectiveness, but it turns out that taking into account only the recently changed code gives better results.

Owing to a large number of the investigated project versions, the results provide a solid empirical evidence regarding the extent of usefulness of the process metrics and they reveal a significant improvement in defect prediction due to some process metrics. In particular, the effect size indicated that the effect of the NDC process metric is substantial 
to achieve better defect prediction which is, in turn, an important contribution of the conducted research. It is worth mentioning that most, if not all, of the related research works do not provide empirical evidence in terms of effect sizes. Effect size proved much more robust empirical evidence than statistical significance alone. This is because significance based on a statistical test does not imply that the result is of practical importance, since even very small differences will be significant if sample sizes are large enough Rosenthal and DiMatteo (2001). Therefore, studies that rely on significance levels may be misleading and it is important to use effect sizes. As a result, our important contribution is that the NDC process metric is of practical importance to achieve better defect prediction.

Furthermore, the paper includes detailed guidelines how to estimate practical importance via effect size measures in case of both parametric and nonparametric analyses which is unique in software defect prediction literature.

We may also conclude that process metrics constitute a different source of information than the product metrics and that employing process metrics in the defect prediction can lead to better results than working only with the product metrics which is another substantial contribution.

There were differences in the values of most of the process metrics between the opensource and the industrial projects (see Sect. 5.1). That confirms to some extent the findings of Jureczko and Madeyski (2010) who investigated the cross-project defect prediction and found that there are some significant differences between the open-source and the industrial projects. The aforementioned study (Jureczko and Madeyski 2010) examined only the product (size and structure) metrics (i.e., the process metrics were not analyzed). That shows an interesting path for the further research toward clustering software projects with regard to defect prediction, where the process metrics will be used as clustering basis.

We argue that there are important differences between the open-source and the industrial projects which can affect our results. But what are those differences? First obvious difference is related to different development processes which are usually more formal, centralized and defined in detail in industrial environments, while less formal and more decentralized in open-source communities. Second difference comes from the ways to organize development teams (e.g., co-located, outsourced, or global). Co-located teams (a group of people working together in an office) are usually not possible in open-source projects, while widely used in industrial projects. An outsourcing arrangement involves two or more separate teams, with responsibilities divided explicitly between the teams. For example, one team in a company headquarters might be focused on specification, while the other team might be located in "low cost" location and do implementation and testing. This kind of arrangement is also common for industrial projects but not open source ones. Open-source projects are usually developed as global projects everybody with enough skills can join. As a result, we may expect more diverse behavior of developers in the opensource projects than in the industrial ones.

It is also interesting to see how the differences between the open-source and the industrial projects affect our results or their interpretation. An interesting observation is that in all of the releases of industrial projects at least one process metric improved prediction models, which is not the case in almost half of the analyzed releases of opensource projects. It seems to corroborate the aforementioned finding about differences between the open-source and the industrial projects. It also suggests the extent of usefulness of process metrics in both industrial and open source environments which can be seen as another contribution of the conducted research. Our interpretation of the results is that the effectiveness of the process metrics in the industrial projects may be higher than in 
the open-source projects due to the aforementioned differences between the open-source and the industrial projects, which influence the behavior of developers. However, further empirical studies are needed to confirm it.

It would be a great finding to identify process improvement opportunities on a basis of the studied relations of process metrics with defects. The more so that to fail and to learn from failure are essential parts of the engineering discipline (Petroski 1985; Kalinowski et al. 2012). However, causal investigations are not easy in practice because we have to carefully recognize what truly constitutes a "cause." Three conditions must be established in order to demonstrate a causal relationship: (1) there must be a correlation or association between the hypothesized cause and effect, (2) the cause must precede the effect in time, (3) the mechanism linking the cause to the effect must be identified. The first condition implies that when the cause occurs, the effect is also likely to be observed. In fact, we demonstrated that condition through statistical correlation analyses in Sect. 5.1. Hence, it can be seen as a partial contribution of the conducted research also to defect causal analysis (defect prevention), since the studied relations of process metrics with defects may also show process improvement opportunities to prevent defects from occurring in the future.

In order to establish evidence, further studies should be conducted, as the investigation of a greater number of software projects would increase the diversity of investigated data and thus improve the external validity of the research results. Although different techniques (e.g., logistic regression, Naive Bayes, neural networks) are used in the field of defect prediction, it is not obvious whether the factors that work well in one technique will also be useful in other methods. Therefore, the experiments should be replicated using other model creation techniques. The results obtained in this study may be applied in the models which were created using linear regression. In order to decide whether they are applicable in other conditions, further research is necessary.

A number of online resources has been published. Each of them is mentioned in the part of paper to which it refers to. However, to provide the reader with a good overview about them, we list them all here:

- Ckjm, a product metrics calculator developed by Spinellis and Jureczko—http://gromit. iiar.pwr.wroc.pl/p_inf/ckjm/

- BugInfo, a process metrics calculator developed by Jureczko-http://kenai.com/ projects/buginfo

- QualitySpy, tool used to calculate the NML metric, developed by Jureczko, Magott and others-http://java.net/QualitySpy

- Metric Repository-http://purl.org/MarianJureczko/MetricsRepo

- Data set used in this study-http://purl.org/MarianJureczko/MetricsRepo/ WhichMetricsImprovePrediction, http://madeyski.e-informatyka.pl/tools/softwaredefect-prediction/

- Additional details about the experiments-http://purl.org/MarianJureczko/ ProcessMetricsExperiments

- Metrics definitions-http://madeyski.e-informatyka.pl/download/ JureczkoMadeyskiSoftwareProductMetrics

- Projects descriptions—http://madeyski.e-informatyka.pl/download/ JureczkoMadeyskiOpenSourceProjects

Acknowledgments The authors are very grateful to the open-source communities and to the Capgemini Polska, which allowed the analysis of their industrial software projects. As a result, we were able to minimize threats to external validity of our research results. 
Open Access This article is distributed under the terms of the Creative Commons Attribution License which permits any use, distribution, and reproduction in any medium, provided the original author(s) and the source are credited.

\section{References}

Antoniol, G., Ayari, K., Di Penta, M., Khomh, F., \& Guéhéneuc, Y. G. (2008). Is it a bug or an enhancement? A text-based approach to classify change requests. In CASCON'08: Proceedings of the 2008 conference of the center for advanced studies on collaborative research (pp. 304-318) New York, NY: ACM. doi10.1145/1463788.1463819.

Arisholm, E., \& Briand, L. C. (2006). Predicting fault-prone components in a java legacy system. In ISESE'06: Proceedings of the 2006 ACM/IEEE international symposium on empirical software engineering (pp. 8-17). New York, NY: ACM. doi10.1145/1159733.1159738.

Bacchelli, A., D’Ambros, M., \& Lanza, M. (2010). Are popular classes more defect prone? In Rosenblum D, Taentzer G (eds) Fundamental approaches to software engineering, Lecture Notes in Computer Science, (Vol. 6013, pp. 59-73). Berlin/Heidelberg: Springer. doi:10.1007/978-3-642-12029-9_5.

Bansiya, J., \& Davis, C. G. (2002). A hierarchical model for object-oriented design quality assessment. IEEE Transactions on Software Engineering, 28(1), 4-17. doi:10.1109/32.979986.

Basili, V. R., Briand, L. C., \& Melo, W. L. (1996). A validation of object-oriented design metrics as quality indicators. IEEE Transactions on Software Engineering, 22(10), 751-761. doi:10.1109/32.544352.

Bell, R. M., Ostrand, T. J., \& Weyuker, E. J. (2006). Looking for bugs in all the right places. In ISSTA'06: Proceedings of the 2006 international symposium on Software testing and analysis (pp. 61-72). New York, NY: ACM. doi:10.1145/1146238.1146246.

Boehm, B. W., \& Papaccio, P. N. (1988). Understanding and controlling software costs. IEEE Transactions on Software Engineering, 14, 1462-1477. doi:10.1109/32.6191.

Catal, C., \& Diri, B. (2009). A systematic review of software fault prediction studies. Expert Systems with Applications, 36(4), 7346-7354. doi:10.1016/j.eswa.2008.10.027.

Chidamber, S. R., \& Kemerer, C. F. (1994). A metrics suite for object oriented design. IEEE Transactions on Software Engineering, 20(6), 476-493. doi:10.1109/32.295895.

Cook, T. D., \& Campbell, D. T. (1979). Quasi-experimentation: Design and analysis issues. Boston, MA: Houghton Mifflin Company.

D’Ambros, M., Bacchelli, A., \& Lanza, M. (2010a). On the Impact of Design Flaws on Software Defects. In QSIC'10: Proceedings of the 2010 10th international conference on quality software, IEEE computer society (pp. 23-31). Washington, DC. doi:10.1109/QSIC.2010.58.

D’Ambros, M., Lanza, M., \& Robbes, R. (2010b). An extensive comparison of bug prediction approaches. In MSR'10: Proceedings of the 2010 7th IEEE working conference on mining software repositories (pp. 31-41). doi:10.1109/MSR.2010.5463279.

Denaro, G., \& Pezzè, M. (2002). An empirical evaluation of fault-proneness models. In Proceedings of the 24th international conference on software engineering, ICSE'02 (pp. 241-251). New York, NY: ACM. doi:10.1145/581339.581371.

Dunlap, W. P., Cortina, J. M., Vaslow, J. B., \& Burke, M. J. (1996). Meta-analysis of experiments with matched groups or repeated measures designs. Psychological Methods, 1(2), 170-177.

Endres, A., \& Rombach, D. (2003). A handbook of software and systems engineering. Reading: AddisonWesley.

Fenton, N., Neil, M., Marsh, W., Hearty, P., Radlinski, L., \& Krause, P. (2007). Project data incorporating qualitative factors for improved software defect prediction. In ICSEW'07: Proceedings of the 29th international conference on software engineering workshops, IEEE Computer Society, Washington, DC. doi:10.1109/ICSEW.2007.171.

Fenton, N. E., \& Neil, M. (1999). A critique of software defect prediction models. IEEE Transactions on Software Engineering, 25, 675-689, doi10.1109/32.815326, http://dl.acm.org/citation.cfm?id=325392. 325401 .

Fenton, N. E., \& Ohlsson, N. (2000). Quantitative analysis of faults and failures in a complex software system. IEEE Transactions on Software Engineering, 26(8), 797-814. doi:10.1109/32.879815.

Fischer, M., Pinzger, M., \& Gall, H. (2003). Populating a release history database from version control and bug tracking systems. In ICSM'03: Proceedings of the international conference on software maintenance (p. 23). Washington, DC: IEEE Computer Society. doi:10.1109/ICSM.2003.1235403.

Giger, E., Pinzger, M., \& Gall, H. (2011a). Using the gini coefficient for bug prediction in eclipse. In Proceedings of the 12th international workshop on principles of software evolution and the 7th annual 
ERCIM workshop on software evolution, IWPSE-EVOL'11 (pp. 51-55). New York, NY: ACM. doi:10. $1145 / 2024445.2024455$.

Giger, E., Pinzger, M., \& Gall, H. C. (2011b). Comparing fine-grained source code changes and code churn for bug prediction. In Proceedings of the 8th working conference on mining software repositories, MSR'11 (pp. 83-92). New York, NY: ACM. doi:10.1145/1985441.1985456.

Graves, T. L., Karr, A. F., Marron, J. S., \& Siy, H. (2000). Predicting fault incidence using software change history. IEEE Transactions on Software Engineering, 26(7), 653-661. doi:10.1109/32.859533.

Grissom, R. J., \& Kim, J. J. (2012). Effect sizes for research: Univariate and multivariate applications. Taylor and Francis: Routledge.

Gyimothy, T., Ferenc, R., \& Siket, I. (2005). Empirical validation of object-oriented metrics on open source software for fault prediction. IEEE Transactions on Software Engineering, 31(10), 897-910. doi:10. 1109/TSE.2005.112.

Hall, T., Beecham, S., Bowes, D., Gray, D., \& Counsell, S. (2012). A systematic literature review on fault prediction performance in software engineering. IEEE Transactions on Software Engineering, 38(6), 1276-1304. doi:10.1109/TSE.2011.103.

Hassan, A. E. (2009). Predicting faults using the complexity of code changes. In ICSE'09: Proceedings of the 31st international conference on software engineering (pp. 78-88). Washington, DC: IEEE Computer Society. doi:10.1109/ICSE.2009.5070510.

Henderson-Sellers, B. (1996). Object-oriented metrics: Measures of complexity. Upper Saddle River, NJ: Prentice-Hall.

Illes-Seifert, T., \& Paech, B. (2010). Exploring the relationship of a file's history and its fault-proneness: An empirical method and its application to open source programs. Information and Software Technology, 52(5), 539-558. doi:10.1016/j.infsof.2009.11.010.

Jureczko, M., \& Madeyski, L. (2010). Towards identifying software project clusters with regard to defect prediction. In PROMISE'2010: Proceedings of the 6th international conference on predictor models in software engineering (pp. 9:1-9:10). ACM doi:10.1145/1868328.1868342, http://madeyski.einformatyka.pl/download/JureczkoMadeyski10f.pdf.

Jureczko, M., \& Madeyski, L. (2011a). A review of process metrics in defect prediction studies. Metody Informatyki Stosowanej 30(5), 133-145, http://madeyski.e-informatyka.pl/download/Madeyski11.pdf.

Jureczko, M., \& Madeyski, L. (2011b). Open source project descriptions. Report SPR 1/2014, Faculty of Computer Science and Management, Wroclaw University of Technology, http://madeyski.einformatyka.pl/download/JureczkoMadeyskiOpenSourceProjects.pdf.

Jureczko, M., \& Madeyski, L. (2011c). Software product metrics used to build defect prediction models. Report SPR 2/2014, Faculty of Computer Science and Management, Wroclaw University of Technology, http://madeyski.e-informatyka.pl/download/JureczkoMadeyskiSoftwareProductMetrics.pdf.

Jureczko, M., \& Magott, J. (2012), QualitySpy: A framework for monitoring software development processes. Journal of Theoretical and Applied Computer, Science, 6(1), 35-45. www.jtacs.org/archive/ 2012/1/4/JTACS_2012_01_04.

Jureczko, M., \& Spinellis, D. (2010). Using object-oriented design metrics to predict software defects, monographs of system dependability, models and methodology of system dependability (pp. 69-81). Wroclaw, Poland: Wroclaw University of Technology Press. http://www.dmst.aueb.gr/dds/pubs/conf/ 2010-DepCoS-RELCOMEX-ckjm-defects/html/JS10.

Kalinowski, M., Card, D. N., \& Travassos, G. H. (2012). Evidence-based guidelines to defect causal analysis. IEEE Software, 29(4), 16-18, doi:10.1109/MS.2012.72.

Kampenes, V. B., Dybå, T., Hannay, J. E., \& Sjøberg D. I. K. (2007). Systematic review: A systematic review of effect size in software engineering experiments. Information and Software Technology, 49(11-12), 1073-1086. doi:10.1016/j.infsof.2007.02.015.

Khoshgoftaar, T. M., Allen, E. B., Halstead, R., Trio, G. P., \& Flass, R. M. (1998). Using process history to predict software quality. Computer, 31(4), 66-72. doi:10.1109/2.666844.

Kim, S., Zimmermann, T., Whitehead, Jr. E. J., \& Zeller, A. (2007). Predicting Faults from Cached History. In ICSE'07: Proceedings of the 29th international conference on software engineering (pp. 489-498). Washington, DC: IEEE Computer Society. doi:10.1109/ICSE.2007.66.

Kitchenham, B. (2010). What's up with software metrics? A preliminary mapping study. Journal of Systems and Software, 83(1), 37-51. doi:10.1016/j.jss.2009.06.041.

Layman, L., Kudrjavets, G., \& Nagappan, N. (2008). Iterative identification of fault-prone binaries using inprocess metrics. In ESEM'08: Proceedings of the second ACM-IEEE international symposium on empirical software engineering and Measurement (pp. 206-212), New York, NY: ACM. doi:10.1145/ 1414004.1414038 . 
Madeyski, L. (2006). Is external code quality correlated with programming experience or feelgood factor? Lecture Notes in Computer Science (Vol. 4044, pp. 65-74). doi:10.1007/11774129_7 Draft: http:// madeyski.e-informatyka.pl/download/Madeyski06b.pdf.

Madeyski, L. (2010). Test-driven development: An empirical evaluation of agile practice. Heidelberg, Dordrecht, London, New York: Springer, doi:10.1007/978-3-642-04288-1, http://www.springer.com/ 978-3-642-04287-4.

Martin, R. (1994). OO design quality metrics—an analysis of dependencies. In OOPSLA'94: Proceedings of workshop pragmatic and theoretical directions in object-oriented software metrics (pp. 1-8), http:// www.objectmentor.com/resources/articles/oodmetrc.

Matsumoto, S., Kamei, Y., Monden, A., ichi Matsumoto, K., \& Nakamura, M. (2010). An analysis of developer metrics for fault prediction. In PROMISE'10: Proceedings of the sixth international conference on predictor models in software engineering (pp. 18:1-18:9). ACM. doi:10.1145/1868328. 1868356.

McCabe, T. (1976). A complexity measure. IEEE Transactions on Software Engineering, 2, 308-320. doi:10.1109/TSE.1976.233837.

Moser, R., Pedrycz, W., \& Succi, G. (2008). A comparative analysis of the efficiency of change metrics and static code attributes for defect prediction. In ICSE'08: Proceedings of the 30th international conference on software engineering (pp. 181-190). New York, NY: ACM. doi:10.1145/1368088.1368114.

Nagappan, N., \& Ball, T. (2005). Use of relative code churn measures to predict system defect density. In ICSE'05: Proceedings of the 27th international conference on software engineering (pp. 284-292). New York, NY: ACM. doi:10.1145/1062455.1062514

Nagappan, N., \& Ball, T. (2007). Using software dependencies and churn metrics to predict field failures: An empirical case study. In ESEM'07: Proceedings of the first international symposium on empirical software engineering and measurement (pp. 364-373). Washington, DC: IEEE Computer Society. doi:10.1109/ESEM.2007.13.

Nagappan, N., Murphy, B., \& Basili, V. (2008). The influence of organizational structure on software quality: an empirical case study. In ICSE'08: Proceedings of the 30th international conference on software engineering (pp. 521-530). New York, NY: ACM. doi10.1145/1368088.1368160.

Nagappan, N., Zeller, A., Zimmermann, T., Herzig, K., \& Murphy, B. (2010). Change bursts as defect predictors. Technical report, Microsoft Research, http://research.microsoft.com/pubs/137315/bursts.

Ostrand, T. J., \& Weyuker, E. J. (2002). The distribution of faults in a large industrial software system. In ISSTA'02: Proceedings of the 2002 ACM SIGSOFT international symposium on software testing and analysis (pp. 55-64). New York, NY: ACM. doi:10.1145/566172.566181.

Ostrand, T. J., Weyuker, E. J., \& Bell, R. M. (2004). Where the bugs are. In ISSTA'04: Proceedings of the 2004 ACM SIGSOFT international symposium on software testing and analysis (pp. 86-96). New York, NY: ACM. doi:10.1145/1007512.1007524.

Ostrand, T. J., Weyuker, E. J., \& Bell, R. M. (2005). Predicting the location and number of faults in large software systems. IEEE Transactions on Software Engineering, 31(4), 340-355. doi:10.1109/TSE. 2005.49.

Petroski, H. (1985). To engineer is human: The role of failure in successful design. New York: St. Martin's Press.

Purao, S., \& Vaishnavi, V. (2003). Product metrics for object-oriented systems. ACM Computing Surveys, 35(2), 191-221. doi:10.1145/857076.857090.

Purushothaman, R., \& Perry, D. E. (2005). Toward understanding the rhetoric of small source code changes. IEEE Transactions on Software Engineering, 31, 511-526. doi:10.1109/TSE.2005.74.

Ratzinger, J., Pinzger, M., \& Gall, H. (2007). EQ-Mine: Predicting short-term defects for software evolution. In M. Dwyer \& A. Lopes (Eds.), Fundamental approaches to software engineering, Lecture Notes in Computer Science, (Vol. 4422, pp. 12-26) Berlin/Heidelberg: Springer. doi:10.1007/978-3-54071289-3_3.

Rosenthal, R. (1991). Meta-analytic procedures for social research (2nd ed.). Newbury Park, CA: SAGE.

Rosenthal, R., \& DiMatteo, M. R. (2001). Meta-analysis: Recent developments in quantitative methods for literature reviews. Annual Review of Psychology, 52, 59-82. doi:10.1146/annurev.psych.52.1.59.

Schröter, A., Zimmermann, T., Premraj, R., \& Zeller, A. (2006). If your bug database could talk. In Proceedings of the 5th international symposium on empirical software engineering, volume II: Short papers and posters (pp. 18-20).

Shadish, W. R., Cook, T. D., \& Campbell, D. T. (2002). Experimental and quasi-experimental designs for generalized causal inference. Boston, MA: Houghton Mifflin.

Shihab, E., Jiang, Z. M., Ibrahim, W. M., Adams, B., \& Hassan, A. E. (2010). Understanding the impact of code and process metrics on post-release defects: A case study on the Eclipse project. In ESEM'10: 
Proceedings of the 2010 ACM-IEEE international symposium on empirical software engineering and measurement (pp. 1-10). New York, NY:ACM. doi:10.1145/1852786.1852792.

Śliwerski, J., Zimmermann, T., \& Zeller, A. (2005). When do changes induce fixes? In MSR'05: Proceedings of the 2005 international workshop on mining software repositories (pp. 1-5). New York, NY: ACM doi:10.1145/1083142.1083147.

Tang, M. H., Kao, M. H., \& Chen, M. H. (1999). An empirical study on object-oriented metrics. In METRICS'99: Proceedings of the 6th international symposium on software metrics (p. 242). Washington, DC: IEEE Computer Society. doi: 10.1109/METRIC.1999.809745.

Wahyudin, D., Schatten, A., Winkler, D., Tjoa, A. M., \& Biffl, S. (2008). Defect prediction using combined product and project metrics - a case study from the open source "Apache" MyFaces project family. In SEAA'08: Proceedings of the 2008 34th euromicro conference software engineering and advanced applications (pp. 207-215). Washington, DC: IEEE Computer Society. doi:10.1109/SEAA.2008.36.

Weyuker, E. J., Ostrand, T. J., \& Bell, R. M. (2006). Adapting a fault prediction model to allow widespread usage. In PROMISE'06: Proceedings of the 4th International Workshop on Predictor Models in Software Engineering (pp. 1-5). New York, NY: ACM doi:10.1145/857076.857090.

Weyuker, E. J., Ostrand, T. J., \& Bell, R. M. (2007). Using developer information as a factor for fault prediction. In PROMISE '07: Proceedings of the third international workshop on predictor models in software engineering (p. 8). Washington, DC: IEEE Computer Society. doi:10.1109/PROMISE.2007. 14.

Weyuker, E. J., Ostrand, T. J., \& Bell, R. M. (2008). Do too many cooks spoil the broth? Using the number of developers to enhance defect prediction models. Empirical Software Engineering, 13(5), 539-559. doi: $10.1007 / \mathrm{s} 10664-008-9082-8$.

Weyuker, E. J., Ostrand, T. J., \& Bell, R. M. (2010). Programmer-based Fault Prediction. In PROMISE '10: Proceedings of the sixth international conference on predictor models in software engineering (pp. 19:1-19:10). ACM. doi:10.1145/1868328.1868357.

Wohlin, C., Runeson, P., Höst, M., Ohlsson, M. C., Regnell, B., \& Wesslén, A. (2000). Experimentation in software engineering: An introduction. Norwell, MA: Kluwer Academic Publishers.

Zimmermann, T., Premraj, R., \& Zeller, A. (2007). Predicting Defects for Eclipse. In PROMISE '07: Proceedings of the third international workshop on predictor models in software engineering (p. 9), Washington, DC: IEEE Computer Society. doi:10.1109/PROMISE.2007.10.

Zimmermann, T., Nagappan, N., Gall, H., Giger, E., \& Murphy, B. (2009). Cross-project defect prediction: a large scale experiment on data vs. domain vs. process. In ESEC/FSE '09: Proceedings of the 7th joint meeting of the European software engineering conference and the ACM SIGSOFT symposium on the foundations of software engineering (pp. 91-100). New York, NY: ACM. doi:10.1145/1595696. 1595713.

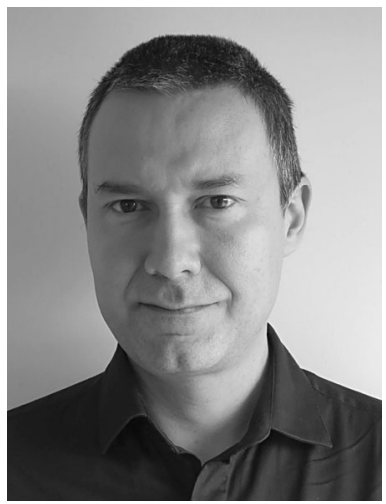

Lech Madeyski received the Ph.D. and Habilitation (D.Sc.) degrees in computer science from the Wroclaw University of Technology, Poland, in 1999 and 2011 respectively. He is currently an Associate Professor at Wroclaw University of Technology, Poland. His research focus is on software quality, mutation testing, empirical (quantitative) research methods (incl. meta-analyses), reproducible research and machine learning in the field of software engineering. He is one of the founders and organizers of the International Conference on Evaluation of Novel Approaches to Software Engineering (ENASE) series which started in 2006 in Erfurt. He is the author of journal papers (e.g. TSE, IST), a book "Test-Driven Development-An Empirical Evaluation of Agile Practice" on empirical evaluation (via statistical analyses and meta-analyses) of Test-Driven Development agile software development practice, published by Springer in 2010. He is a member of ACM and IEEE. 


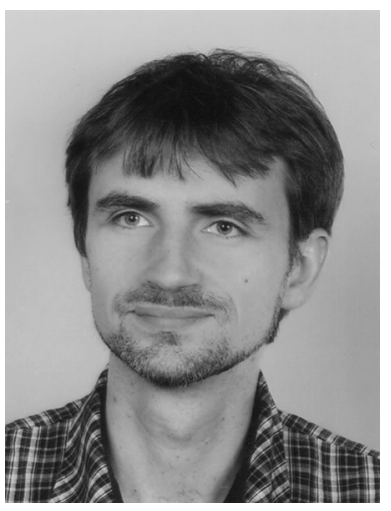

Marian Jureczko received his M.Sc. and Ph.D. degrees in computer science from Wroclaw University of Technology. His main research interests include software quality, software metrics, defect prediction and software testing. $\mathrm{He}$ is currently collaborating closely with industry, he is working as a software engineering for Sii Poland. 